\title{
Value of Office-Based Labs to an Interventional Radiology Practice
}

\author{
Shimwoo Lee ${ }^{1}$ Ravi N. Srinivasa ${ }^{1} \quad$ Prital Patel $^{1} \quad$ Scott Genshaft ${ }^{1}$ Dieter R. Enzmann ${ }^{2}$ \\ ${ }^{1}$ Department of Interventional Radiology, David Geffen School of \\ Medicine at UCLA, Los Angeles, California, United States \\ 2 Department of Radiological Sciences, David Geffen School of \\ Medicine at UCLA, Los Angeles, California, United States \\ Address for correspondence Shimwoo Lee, MD, Department of \\ Interventional Radiology, David Geffen School of Medicine at UCLA, \\ 757 Westwood Plaza, Suite 1638, Los Angeles, CA 90095, United \\ States (e-mail: ShimwooLee@mednet.ucla.edu).
}

J Clin Interv Radiol ISVIR 2023;7:15-19.

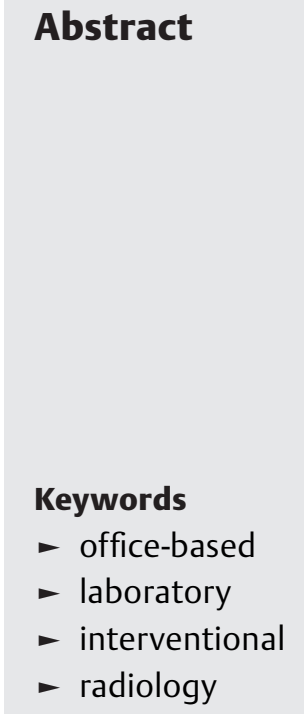

Purpose Office-based laboratories (OBLs), or outpatient interventional suites, are rapidly gaining traction with both patients and interventionalists. Compared with hospitals, OBLs can provide greater convenience for patients, allow physicians greater autonomy, and potentially generate more revenue under a lower cost structure. The aim of this article is to discuss logistics and finances of running an OBL using the authors' institution as a guide for interventional radiologists seeking to establish their own OBL.

Materials and Methods Interventional radiology (IR) procedures performed at an OBL from the authors' academic institution were reviewed from January 2017 to December 2020. Yearly revenues and expenses related to IR procedures were recorded. Results The number and complexity of IR procedures performed in the OBL increased over time with nearly 10-fold growth of revenue from 2017 to 2020.

Conclusion Most IR procedures performed in the hospital are feasible in the OBL setting, and by increasing patient volume and variety over time, an individual OBL can develop into a robust patient care center capable of generating revenue.

\section{Introduction}

The trend toward performing minimally invasive procedures in an outpatient office-based setting is rapidly growing. Over 700 office-based laboratories (OBLs) currently exist in the United States. ${ }^{1}$ This movement has been possible due to technological advances that have allowed physicians to safely perform most endovascular and other interventional procedures in the OBL setting. The advantages of moving out of the conventional hospital setting to outpatient venues are manifold, including increased convenience for patients, greater efficiency, and flexibility for physicians in providing care. Moreover, OBLs provide an opportunity to generate revenue in a growing market.

Medicare and many insurance payers reimburse procedures performed in OBLs at a different, sometimes higher rate, called the "global" fee. ${ }^{2}$ This rate combines payments for both professional and technical services because the overhead expense, including staffing and equipment, is paid for by the physician's office. In contrast, physicians performing procedures in hospital settings receive only professional fees. article published online February 25, 2022
DOI https://doi.org/

$10.1055 / \mathrm{s}-0042-1742729$. ISSN 2457-0214.
(C) 2022. Indian Society of Vascular and Interventional Radiology. All rights reserved.

This is an open access article published by Thieme under the terms of the Creative Commons Attribution-NonDerivative-NonCommercial-License, permitting copying and reproduction so long as the original work is given appropriate credit. Contents may not be used for commercial purposes, or adapted, remixed, transformed or built upon. (https://creativecommons.org/ licenses/by-nc-nd/4.0/)

Thieme Medical and Scientific Publishers Pvt. Ltd., A-12, 2nd Floor, Sector 2, Noida-201301 UP, India 
With recent decreases in reimbursement for professional fees, a well-managed OBL provides an opportunity to create a consistent revenue stream and net earnings if expenses are well controlled.

The global fee for outpatient procedures performed in an OBL may be roughly five times the professional revenue for outpatient cases performed in the hospital settings. ${ }^{3}$ At the same time, there is also significant cost savings to Medicare when procedures are performed in the office. Much of the existing data relates to OBL-based cardiology and vascular surgery practices; literature on office-based procedures in interventional radiology (IR) is relatively sparse. The aim of this article is to discuss logistics and benefits of running an IR-OBL using the authors' institution as an example.

\section{Materials and Methods}

This study received approval from the institutional review board (IRB \#20-001749). The OBL examined in this study is owned by the authors' academic institution. Total cases done in the OBL were identified and records were reviewed for IR cases since inception from January 2017 to December 2020. The number and types of procedures were recorded. The estimated yearly revenues and expenses related to IR procedures were supplied by the institution's financial department. These figures were averaged to tabulate rough reimbursements from various IR procedures.

At a typical OBL in the authors' practice, roughly 8 to 10 patients are treated daily in addition to 3 to 5 patients seen in clinic. On a given workday, the OBL is staffed by one boardcertified fellowship-trained interventional radiologist, two radiology technologists, and three registered nurses. A nurse practitioner performs relatively simple procedures such as paracentesis/thoracentesis. Both physicians and support staff rotate through the main hospital on a weekly basis to maintain varied skillsets.

The typical center is equipped with one angiography suite, one ultrasound room, and one computed tomography (CT) room. The ultrasound and CT rooms are used for diagnostic imaging when not needed for an interventional procedure. Two to three preoperative and recovery beds are available for patients in addition to a dedicated IR clinic room for consultations. Nearby hospitals are made aware of the existence of the facility in the event of emergencies that necessitate higher level of care.

Nearly all procedures are performed in an hour or less. Procedures are performed under moderate sedation and local anesthesia without an anesthesiologist. Patients are monitored by registered nurses before, during, and after procedure. Patients recover in the adjacent recovery bays and are usually discharged within 1 to 2 hours. An attempt is made to follow up every patient by telephone on the next working day. For more complex therapeutic procedures, patients are formally evaluated prior to procedure in clinic and subsequently followed up in clinic as needed to address any complications. Tele-video visits made during the coro- navirus disease 2019 pandemic are likely to play an increasing role in follow-up.

\section{Results}

A diverse number of IR procedures typically performed in the hospital setting were performed in the OBL. The most common procedures in the office include ultrasound- and CT-guided biopsies and drainages, fluoroscopy- and ultrasound-guided joint injections, and central venous catheter placement and removal (-Table 1). Ultrasound-guided thoracentesis and paracentesis, performed by nurse practitioners, were also among the most common procedures.

The more complex, higher revenue procedures included endovenous laser ablations for varicose veins, uterine fibroid embolization, prostate artery embolization, genicular artery embolization, kyphoplasty, hepatic artery embolization including radioembolization and chemoembolization, and peripheral arterial angioplasty and stenting. The average percentage of reimbursement was $48 \%$ (range: $23-73 \%$ ) with commercial payors and 21\% (range: 16-25\%) with Medicare. Of note, these figures are most likely to vary considerably with region, practice patterns, case mix, and payor types.

Since inception, the OBL has been enjoying steady growth in patient volume, with nearly a threefold rise from 2017 to 2020 (-Fig. 1). The variety and complexity of patients have also increased over time. The percentage of therapeutic interventions that involve a formal consultation before and after procedure (e.g., uterine fibroid embolization, sclerotherapy, and peripheral arterial interventions) has grown fivefold from 2017 to 2020. The variety of vascular procedures has increased over time (-Fig. 2). Port and central venous catheter placement and removals were the vast majority of vascular procedures performed (95\%) in 2018; these procedures still remain the most common (70\%), but the number of more complex procedures, such as varicose vein laser/sclerotherapy (27\%), genicular artery embolizations (15\%), and uterine fibroid embolization (7\%), have expanded in 2020 . The revenue generated by the OBL has grown substantially, nearly 10 fold from 2017 to 2020 (-Fig. 3). The overhead cost of medical supplies and equipment has also risen over time but at a much lower rate. The OBL took $\sim 2$ years to break even in terms of revenue.

Complication rates from were examined for procedures listed as "vascular" in - Table 1 from 2017 to 2020. The percentage of cases with available follow-up data in the electronic medical chart was $84 \%$. The 30 -day postprocedure mortality rate was $0 \%$, and there were no instances of OBL procedures needing hospital transfer for emergency back-up management. The rate of complications resulting in emergency room or specialty care (e.g., wound care) visits was 7\%. These include postuterine embolization pain, radial access site pain, elevated liver enzymes posthepatic embolization, postsclerotherapy cellulitis, postlaser venous therapy skin ulceration, and numbness. All 
Table 1 Types of office-based procedures performed

\begin{tabular}{|c|c|}
\hline Nonvascular & Vascular \\
\hline $\begin{array}{l}\text { - Biopsy (e.g., lung, liver, kidney, pelvis, bone, bone marrow, } \\
\text { lymph node, thyroid) } \\
\text { - Joint injections (for arthrograms and corticosteroid } \\
\text { treatment) } \\
\text { - Drainage catheter placement and removal } \\
\text { - Nerve block (e.g., lumbar facet, epidural) } \\
\text { - Saline infusion sonohysterogram } \\
\text { - Gastrostomy and gastrojejunostomy tube placement } \\
\text { - Nephrostomy tube placement and exchange } \\
\text { - Suprapubic catheter placement } \\
\text { - Peritoneal dialysis catheter placement } \\
\text { - Cholecystostomy exchange and removal } \\
\text { - Biliary tube exchange } \\
\text { - Prostate biopsy, MRl-guided } \\
\text { - Paracentesis and thoracentesis }\end{array}$ & $\begin{array}{l}\text { - Central venous access placement and removal } \\
\text { (e.g., dialysis catheter, port, PICC) } \\
\text { - Endovenous laser ablation } \\
\text { - Transjugular liver biopsy } \\
\text { - Sclerotherapy (e.g., lymphatic malformation, varicose } \\
\text { veins) } \\
\text { - IVC filter placement, retrieval } \\
\text { - Uterine fibroid embolization } \\
\text { - Peripheral arterial angioplasty } \\
\text { - Genicular artery embolization } \\
\text { - Prostate artery embolization } \\
\text { - Hepatic artery embolization (Y-90) } \\
\text { - Kyphoplasty } \\
\text { - Dialysis fistulagram } \\
\text { - Renal artery angioplasty } \\
\text { - Varicocele embolization } \\
\text { - Lymphangiogram }\end{array}$ \\
\hline
\end{tabular}

Abbreviations: IVC, inferior vena cava; MRI, magnetic resonance imaging; PICC, peripherally inserted central catheter. ${ }^{a}$ Majority performed by nurse practitioners.

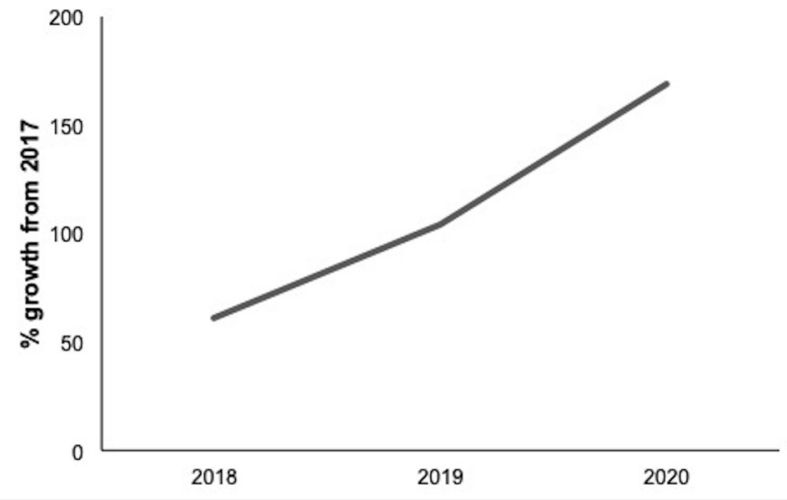

Fig. 1 The percent growth of procedures performed annually in the office-based laboratory during 2018 to 2020 is compared with its baseline year in 2017.

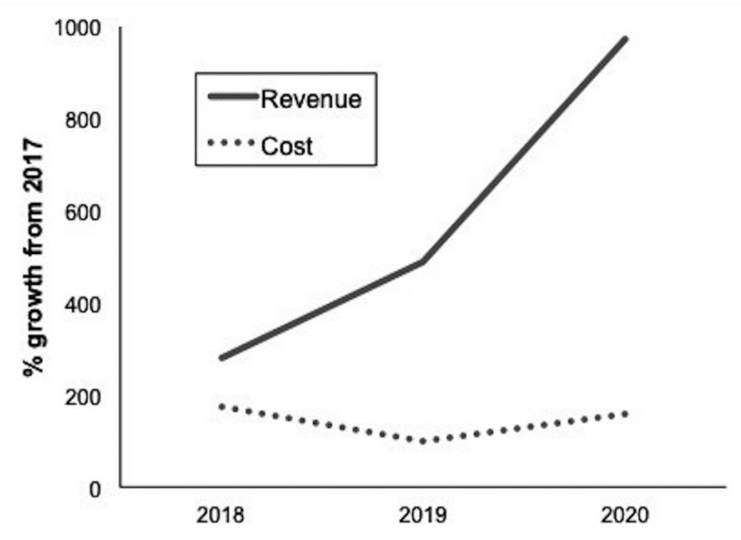

Fig. 3 The percent growth in yearly revenue and percent cost in the office-based laboratory during 2018 to 2020 is compared with its baseline year in 2017.
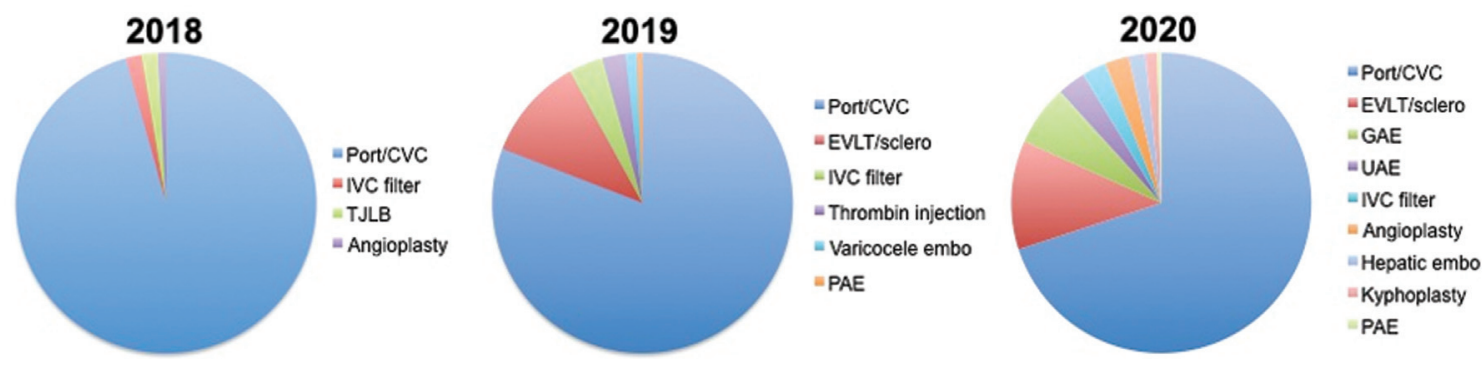

Fig. 2 The proportion of each vascular procedure type performed in the office-based laboratory is depicted in pie chart form by year.

Angioplasty, peripheral artery angioplasty and stenting; EVLT/sclero, endovenous laser treatment and sclerotherapy for varicose veins; GAE, genicular artery embolization; hepatic embo, Yttrium-90 hepatic artery embolization; IVC filter, inferior vena cava filter placement and retrieval; PAE, prostate artery embolization; port/CVC, port and central venous catheter placement and removal; TJLB, transjugular liver biopsy; UFE, uterine artery embolization. 
of these complications resolved with conservative management.

\section{Discussion}

OBLs can overcome several challenges that confront interventional radiologists working in the hospital setting. Interventionalists who work in hospitals may often experience slow turn-around time for procedure rooms, which in turn result in slowed patient care, while facing decreasing reimbursement for their professional fees. On the other hand, OBLs enable physicians to take control of the entire workflow from initial patient greeting to the postprocedural care, allowing for a more streamlined approach for patient care, therefore decreasing turnover time and boosting patient satisfaction. As office-based procedures are reimbursed in global payments, the potential revenue from running an efficient OBL scales nicely with the fixed costs.

\section{Logistics}

To set up an OBL, an interventional radiologist may partner with a business manager to develop a business plan. In an academic setting, the OBLs may be set up as joint venture with the academic institution, as in the authors' case. The cost of initially setting up an OBL largely depends on the availability of a building. The basic requirement is an endovascular suite with a recovery area somewhere in the range of 3,000 square feet. ${ }^{3}$ In addition, a front desk waiting area and office for clinic visits are needed. An estimated two to three million dollars of capital investment are required for equipment such as the angiography suite, CT scanner, ultrasound machine, laser or thermal ablation machine, power injector, and patient monitoring equipment. The center must be stocked with angiographic equipment such as catheters, stents, sheaths, guidewires, and embolic materials using estimates of expected patient volume.

Adequate staffing with skilled personnel is crucial. Radiology technologists and registered nurses, as well as front desk personnel and billing personnel are needed. Protocols for quality control, sterilization, radiation safety, sedation, and aseptic technique must be in place. Postprocedural follow-up of patients must be established to deal with complications in a timely fashion.

\section{Benefits}

OBLs offer numerous advantages for both patients and treating physicians. The office setting can provide a more comfortable patient-focused environment compared with hospitals. Patients can be scheduled faster with greater flexibility and can receive treatment closer to home. This is particularly beneficial for patients living far from major hospitals located in high-traffic urban locations. The OBLs in the authors' practice are located in suburban areas, $\sim 20$ to 30 miles away from the main academic hospital. Continuity of care from familiar staff in subsequent visits adds a personal touch to patient comfort.

Physicians may equally enjoy higher work satisfaction with less likelihood of burnout in the office setting thanks to a more controlled work schedule and greater efficiency. ${ }^{4}$ More procedures can be done during working hours when the office is open compared with similar periods of time in the hospital. Recently, Hickey et al and our experience have shown that establishing an Yttrium-90 radioembolization program in the OBL is not only feasible but also can be more efficient compared with the hospital setting. ${ }^{5}$ This service line requires the presence of a radiopharmacy, or "hot lab" in the OBL to dispense and store radiopharmaceuticals. Procedures conventionally performed in hospitals, therefore, are increasingly done in the office. In the authors' practice, we have generally found transarterial procedures, including liver-directed therapy, prostate artery embolization, and uterine artery embolizations, especially rewarding in the OBL setting. The safety and efficacy of these procedures in the outpatient setting are well supported in the literature. ${ }^{6-8}$ In addition, the authors have found success with venous ablations and peripheral artery disease treatments. Of note, prescreening and selection of less complicated cases for peripheral artery disease are recommended for OBLs.

An additional strength of an OBL is that it can generate referrals. While seeing patients in clinic or reviewing their images, interventional radiologists may identify additional opportunities for providing necessary therapy for patients. For instance, a patient who presents for a liver mass biopsy may later need a port placement, an ablation, or a transarterial therapy. Patients requiring procedures of higher complexity are carefully evaluated first in the OBL clinic and referred to the main hospital if the procedure is deemed too complex to perform in the OBL. If there were concern that a procedure could not be performed with moderate sedation alone, then it would be scheduled with anesthesia in the hospital. As such, OBLs can help generate more volume for the hospital as well.

Educating patients and referring physicians on the wide variety of treatments that an OBL can offer is key to continual growth of patient volume. It may be of particular benefit for an OBL to be a part of a multispecialty office-based practice since physicians within the practice are more likely to refer patients to interventional radiologists whom they are familiar with and trust For instance, the OBL in the authors' institution is part of a multispecialty academic practice, and its typical referral base includes other physician within the group as well as surrounding local primary care and specialty care providers, including nephrologists, oncologists, gynecologists, urologists, and podiatrists.

\section{Potential Pitfalls}

Operating an OBL can come with significant financial risk and is unlikely to succeed without a sound business plan and proactive patient recruitment. There is also a potential for physicians to misuse office-based settings and over-treat patients to achieve higher revenue. ${ }^{8}$ Internal review within a physician group can deter these activities. Although there is data to support the overall safety of office-based procedures in the vascular surgery literature, ${ }^{9}$ more studies establishing safety of performing specific IR procedures are needed. 


\section{Conclusion}

An OBL is a rapidly growing IR practice model that can provide greater convenience for patients, allow physicians greater autonomy, and potentially generate more revenue under a lower cost structure.

Funding

This paper received no financial sources of support, including grants, funding, or other assistance, public or private.

\section{Conflict of Interest}

R.N.S. reports personal fees and other from Boston Scientific, other from Medtronic, other from NXT Biomedical, outside the submitted work. The rest of the coauthors declare no conflict of interest.

\section{References}

1 OEIS. Outpatient Endovascular and Interventional Society. Accessed January 24, 2022 from: https://oeisociety.com
2 Kho H. Financial considerations for office-based intervention labs. Endovasc Today. 2014(Jan):55-58

3 Jain KM, Munn J, Rummel M, Vaddineni S, Longton C. Future of vascular surgery is in the office. J Vasc Surg 2010;51(02):509-513, discussion 513-514

4 Bundy JJ, Hage AN, Srinivasa RN, et al. Burnout among interventional radiologists. J Vasc Interv Radiol 2020;31(04): 607-613.e1

5 Hickey RM, Maslowski JM, Aaltonen ET, et al. Yttrium-90 radioembolization in the office-based lab. J Vasc Interv Radiol 2020;31 (09):1442-1448

6 Bagla S, Piechowiak R. Prostate artery embolization in an outpatient based lab (OBL): safety and radiation exposure. J Vasc Interv Radiol 2017;28:S110

7 Siskin GP, Stainken BF, Dowling K, Meo P, Ahn J, Dolen EG. Outpatient uterine artery embolization for symptomatic uterine fibroids: experience in 49 patients. J Vasc Interv Radiol 2000;11 (03):305-311[Internet]

8 McKeown LA. Endovascular office-based labs: balancing upsides against potential for abuse. TCTMD 2019(Nov):1-3

9 Jain K, Munn J, Rummel MC, Johnston D, Longton C. Office-based endovascular suite is safe for most procedures. J Vasc Surg 2014; 59(01):186-191 